\title{
Effect of sec-O-glucosylhamaudol on mechanical allodynia in a rat model of postoperative pain
}

\author{
Gi-Ho Koh ${ }^{1}$, Hyun Song ${ }^{2}$, Sang Hun Kim ${ }^{2,3}$, Myung Ha Yoon ${ }^{4}$, Kyung Joon Lim²,3, Seon-Hee $\mathrm{Oh}^{5}$, \\ and Ki Tae Jung ${ }^{2,3}$ \\ ${ }^{1}$ Department of Anesthesiology and Pain Medicine, Asan Medical Center, University of Ulsan College of Medicine, Seoul, \\ ${ }^{2}$ Department of Anesthesiology and Pain Medicine, Chosun University Hospital, Gwangju, \\ ${ }^{3}$ Department of Anesthesiology and Pain Medicine, School of Medicine, Chosun University, Gwangju, \\ ${ }^{4}$ Department of Anesthesiology and Pain Medicine, Medical School, Chonnam National University, Gwangju, \\ ${ }^{5}$ School of Medicine, Chosun University, Gwangju, Korea
}

Background: This study was performed in order to examine the effect of intrathecal sec-O-glucosylhamaudol (SOG), an extract from the root of the Peucedanum japonicum Thunb., on incisional pain in a rat model.

Methods: The intrathecal catheter was inserted in male Sprague-Dawley rats $(n=55)$. The postoperative pain model was made and paw withdrawal thresholds (PWTs) were evaluated. Rats were randomly treated with a vehicle (70\% dimethyl sulfoxide) and SOG (10 $\mu \mathrm{g}, 30 \mu \mathrm{g}, 100 \mu \mathrm{g}$, and $300 \mu \mathrm{g})$ intrathecally, and PWT was observed for four hours. Dose-responsiveness and ED50 values were calculated. Naloxone was administered 10 min prior to treatment of SOG $300 \mu \mathrm{g}$ in order to assess the involvement of SOG with an opioid receptor. The protein levels of the $\delta$-opioid receptor, $\kappa$-opioid receptor, and $\mu$-opioid receptor (MOR) were analyzed by Western blotting of the spinal cord.

Results: Intrathecal SOG significantly increased PWT in a dose-dependent manner. Maximum effects were achieved at a dose of $300 \mu \mathrm{g}$ at $60 \mathrm{~min}$ after SOG administration, and the maximal possible effect was $85.35 \%$ at that time. The medial effective dose of intrathecal SOG was $191.3 \mu \mathrm{g}$ (95\% confidence interval, 102.3-357.8). The antinociceptive effects of SOG (300 $\mu \mathrm{g})$ were significantly reverted until $60 \mathrm{~min}$ by naloxone. The protein levels of MOR were decreased by administration of SOG.

Conclusions: Intrathecal SOG showed a significant antinociceptive effect on the postoperative pain model and reverted by naloxone. The expression of MOR were changed by SOG. The effects of SOG seem to involve the MOR. (Korean J Pain 2019; 32: 87-96)

Key Words: Analgesia; Blotting, western; Dimethyl sulfoxide; Hyperalgesia; Nociceptive pain; Pain, postoperative; Rats; Receptors, opioid; Spinal cord; 11-hydroxy-sec-O-glocosylhamaudol.

Received January 14, 2019. Revised February 28, 2019. Accepted March 5, 2019.

Correspondence to: Ki Tae Jung

Department of Anesthesiology and Pain Medicine, School of Medicine, Chosun University, 365 Pilmun-daero, Dong-gu, Gwangju 61453, Korea

Tel: +82-62-220-3223, Fax: +82-62-223-2333, E-mail: mdmole@chosun.ac.kr

ORCID: https://orcid.org/0000-0002-2486-9961

Gi-Ho Koh's current affiliation: Department of Anesthesiology and Pain Medicine, Chosun University Hospital, Gwangju, Korea

(a) This is an open-access article distributed under the terms of the Creative Commons Attribution Non-Commercial License (http:// creativecommons.org/licenses/by-nc/4.0/), which permits unrestricted non-commercial use, distribution, and reproduction in any medium, provided the original work is properly cited.

(C) The Korean Pain Society, 2019 


\section{INTRODUCTION}

Even with the adequate use of medication, approximately $40 \%$ of patients experience moderate to severe postoperative pain [1,2]. Postoperative pain is a form of acute pain that is directly or indirectly involved in the occurrence of postoperative complications [2,3]. Surgical insults, such as incision tissue injuries, directly activate the peripheral nociceptors, resulting in an increase of central neuronal excitability, and leading to peripheral and central sensitization as a consequence [2,4]. Prolonged sensitization caused by inadequate analgesia may result in chronic postoperative pain or allodynia. These pathologic states may result in the decrease of the functions of the lung, heart, digestive system, and urological system, and can even cause psychological effects such as insomnia, depression, and anxiety in postoperative patients [3-5]. Accordingly, the effective mitigation of postoperative pain should be done as quickly as possible in order to reduce pain, promote recovery, and prevent complications [6]. Active postoperative pain management is an essential component of the care of surgical patients in the prevention of the development of chronic pain disorder. However, despite an increase in related scientific evidence, postoperative pain management remains insufficient because many patients still suffer from severe pain after surgery [6]. Thus, finding effective medications for postoperative analgesia is still necessary.

Substances derived from natural products have been used to treat pain disorders for a long time [7]. Discovering new chemical entities for postoperative pain from natural products can be an opportunity to improve the quality of life for the patient. The root of the Peucedanum japonicum Thunb., distributed throughout Japan, Philippines, China, and Korea has been traditionally used as not only a food but also as an herbal medicine for cough, cold, headaches, and neuralgic disease $[8,9]$. It has been recognized that substances derived from the Peucedanum species show antiplatelet aggregation [9], antioxidant activity [10], antiinflammatory activity [11], and inhibitory activity on cyclooxygenase (COX) 1 and 2 [12]. According to studies on the isolated constituents of Peucedanum japonicum Thunb., sec-O-glucosylhamaudol (SOG) shows analgesic activity $[12,13]$. In particular, a recent study showed that intrathecal SOG administration has an antinociceptive effect in a formalin test on a rat model, and shows the possibility of the involvement of SOG on opioid receptors [14].

Thus, we hypothesized that SOG exerts analgesic effects on postoperative incisional pain. We conducted the present study in order to evaluate the effect of SOG on mechanical allodynia in a rat model of postoperative pain in association with opioid receptors, and to set a clinical foundation for the use of SOG as a medication from natural products for postoperative pain control.

\section{MATERIALS AND METHODS}

\section{Animals preparation and intrathecal catheterization}

Following approval from the Institutional Animal Care and Use Committee of Chonnam National University (CNU IACUC-H-2017-82), this study was conducted in compliance with guidelines from the International Association for the Study of Pain on ethical standards for the investigation of experimental pain in animals (Fig. 1) [15].

Male Sprague-Dawley rats weighing 200-250 g were used for the experiments $(n=55)$. Rats were each housed in cages in the animal facility under constant temperature $\left(22^{\circ} \mathrm{C} \pm 0.5^{\circ} \mathrm{C}\right)$, a $12-\mathrm{h}$ light/dark cycle, and food and water available ad libitum. For the administration of drugs, intrathecal catheterization was done with sevoflurane anesthesia [16]. Each rat was fixed in a stereotaxic apparatus, and a longitudinal incision was made on the atlantooccipital membrane following sterile dressing for the exposure of the cisterna magna. An intrathecal catheter (polyethylene-5) was implanted via the cisterna magna and was advanced caudally by $8.5 \mathrm{~cm}$ so as to place the proximal portion of catheter on the level of lumbar enlargement. The distal portion of the catheter was secured to the skin of the head and anchored firmly by suture. The skin was sutured with a 3-0 silk and the end of the externalized catheter was closed up with a 30-gauge stainless steel wire after confirming the leakage of cerebrospinal fluid through the catheter. Following intrathecal catheter implantation, general anesthesia was stopped, and rats were kept in individual cages in order to observe the return of ambulatory functions after they awoke from anesthesia. Rats with signs of neurologic dysfunction such as limping or sensory deficits were immediately euthanized with volatile anesthetics. Rats without neurologic deficits were sent back to the vivarium and housed in individual cages for a week. 


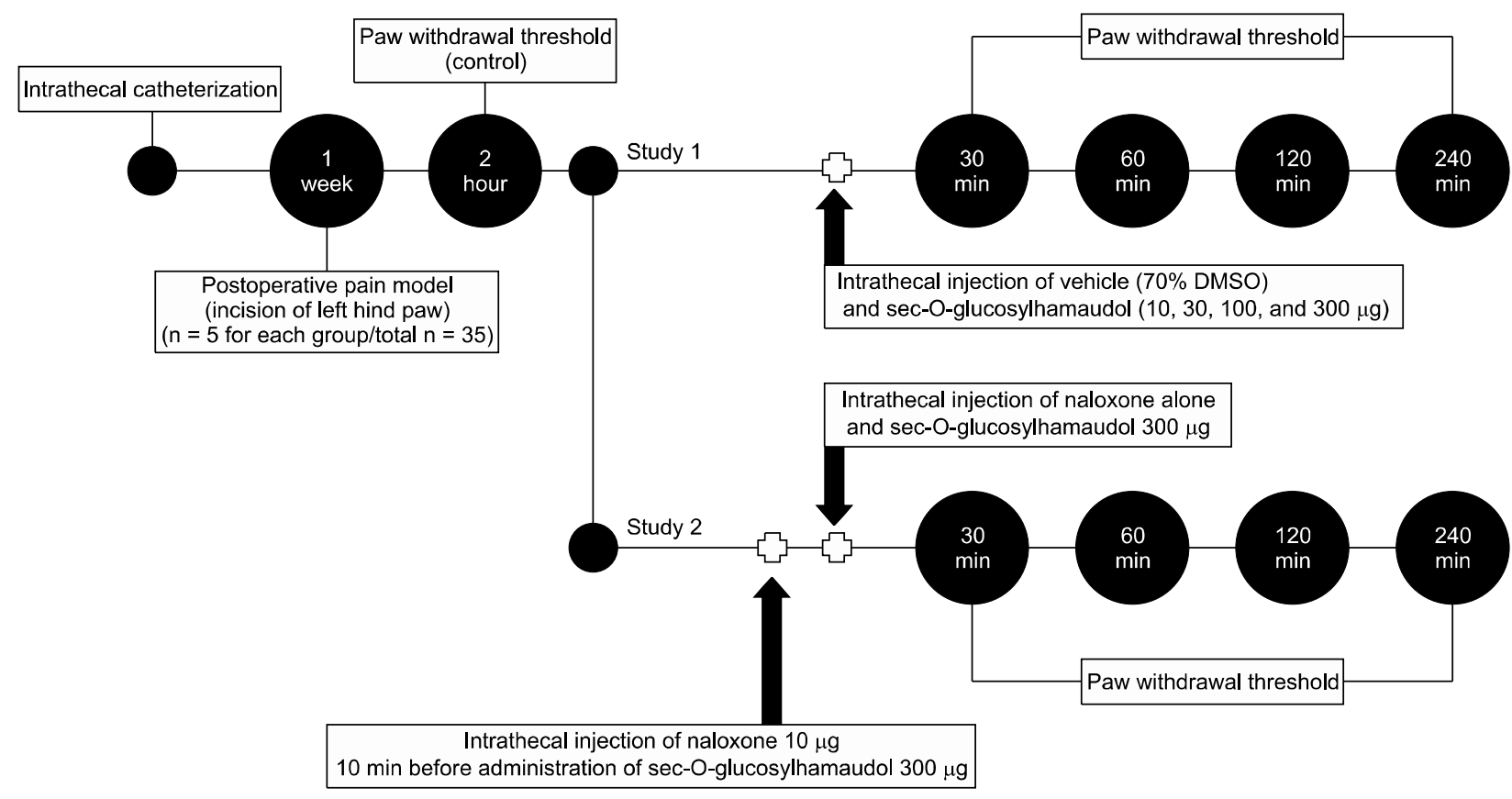

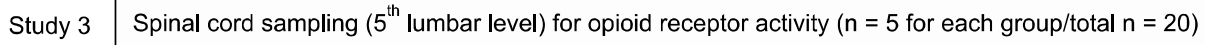

Group 1: naïve
Group 2: control (2 hours after incision of left hind paw)
Group 3: 1 hour after vehicle administration (70\% DMSO)
Group 4: 1 hour after sec-O-glucosylhamaudol $300 \mu \mathrm{g}$ administration

Fig. 1. Diagram illustrating the progress of the study protocol throughout the experiment. DMSO: dimethyl sulfoxide.

2. Postoperative pain model and behavioral study for postincisional mechanical allodynia

A week after intrathecal catheterization, rats were assessed for whether sensory abnormality had developed after intrathecal catheterization. Only rats without any neurological abnormality were used for this study. A postoperative pain model was applied according to the method outlined by Brennan et al. [17]. Anesthesia was conducted with sevoflurane, and the plantar surfaces of the left hind paws were prepared for incision. Following sterilization, a $1 \mathrm{~cm}$ longitudinal incision was made from $0.5 \mathrm{~cm}$ distal to the proximal edge of the heel towards the toes. Skins and fasciae were incised in order to expose the plantaris muscle. The plantaris muscle was then elevated and incised longitudinally with the muscle origin and insertion points kept intact. Gentle pressure was applied to control the bleeding and the incised skin was sutured with a 4-O silk. After dressing with povidone-iodine solution, rats were sent back to cages for recovery.
Two hours after the incision of the hind paw, an initial test was conducted in order to confirm the development of postoperative pain by assessing the occurrence of mechanical allodynia and to measure the control value of the postincisional paw withdrawal threshold (PWT). The PWT was measured in response to mechanical stimulation using von Frey filaments (Stoelting, Wood Dale, IL, USA). After acclimation in the laboratory environment for $30 \mathrm{~min}$, the hind paws of the rats were accessed via apertures created in the mesh floor of the cage. Mechanical stimulation was applied to the plantar surface of the hind paw vertically for $5 \mathrm{~s}$ with a series of eight von Frey filaments (0.4, 0.7, $1.2,2.0,3.6,5.5,8.5$, and $15 \mathrm{~g}$ ). After applying the von Frey filaments to the adjacent edge of the wound in the incised hind paw or to the center of the plantar surface of the non-injured paw, abrupt withdrawal or a characteristic flinching response of the hind paw after the stimulation of a filament was considered a positive response. The response was measured two times for each filament caliber with about a 3-min stimulation-free period in- 
between. The PWT was calculated by the up and down method [18]. The cut-off value was a negative response to $15 \mathrm{~g}$ and only rats showing marked mechanical allodynia (PWT $<5 \mathrm{~g}$ ) were included for the study.

\section{Drug preparations}

SOG (purity > 95\%) was purchased from the Natural Product Bank (Gyeongsan, Korea). Diluted solutions of SOG were prepared at 10,30,100, and $300 \mu \mathrm{g}$ after dissolving in $70 \%$ dimethyl sulfoxide (DMSO) as $10 \mu \mathrm{l}$ solutions.

\section{Experimental design and administration of intrathecal SOG}

The total number of rats used was 35, with 5 rats per dilution of SOG (Fig. 1). After confirmation of mechanical allodynia through achievement of the incisional pain model, intrathecal administration of SOG preparations (as $10 \mu \mathrm{l}$ solutions) or a vehicle (10 $\mu \mathrm{l}$ of $70 \%$ DMSO) were conducted using a hand-driven, gear-operated syringe pump. Then, an additional $10 \mu \mathrm{l}$ of $70 \%$ DMSO was administered to flush the SOG to the intrathecal space. Intrathecal administration of the experimental drug was randomly performed by computer-generated numbers. The effects of intrathecal SOG of each dilution on the PWT were measured for $4 \mathrm{~h}$ by other researcher. The PWT measured $120 \mathrm{~min}$ after incision was regarded as the control postincision threshold. A series of tests were then conducted prior to incision and 30, 60, 120, and 240 min after delivery of the drug.

\section{Dose-responsiveness and ED50 of SOG}

Dose-responsiveness and median effective analgesic dose (ED50) values were calculated. Dose-response data of the intrathecal SOG were calculated as the percentages of the maximum possible effect (\%MPE) as follows:

$\% \mathrm{MPE}=\left[\frac{\text { post-drug PWT }- \text { pre-drug control PWT }}{\text { pre-incision PWT }- \text { post-incision control PWT }}\right] \times 100$

The ED 50 of SOG and its confidence interval were calculated using a standard linear regression analysis of a dose-response curve, according to the method outlined by Tallarida and Murray [19].
6. Reversal of the effect of intrathecal SOG by opioid antagonist

A further experiment was conducted in order to determine the involvement of SOG on the opioid receptor. A $10 \mu \mathrm{g}$ dose of naloxone (Tocris Cookson, Avonmouth, UK) was administered intrathecally $10 \mathrm{~min}$ prior to intrathecal injection of SOG $300 \mu \mathrm{g}$, which showed a maximal antinociceptive effect. The PWT was measured in the same time sequence as that of the prior study.

\section{Western blotting analyses for opioid receptor protein levels}

For the analysis of opioid receptor protein levels, Western blotting analyses were performed on the spinal cord samples from the rats. The spinal cord samples were obtained from the rats 60 min after administration of SOG, when it shows the strongest effects. Rats were divided into five groups: Group 1, naïve; Group 2, control (2 h after incision of the left hind paw); Group 3, 1 h after vehicle administration (70\% DMSO); and Group 4, 1 h after SOG 300 $\mu \mathrm{g}$ administration. Rats were euthanized and decapitated under sevoflurane anesthesia, and the spinal cord was isolated by flushing with ice-cold phosphate-buffered saline through an 18-gauge needle which was inserted into the caudal end of the vertebral column. The ipsilateral dorsal spinal cord at L5 was then isolated and stored at $-70^{\circ} \mathrm{C}$, using liquid nitrogen, immediately until homogenization.

Tissue samples ( $\mathrm{n}=5$ for each group, total $\mathrm{n}=20$ ) were dissected from frozen specimens and homogenized in a lysis buffer with a Dounce homogenizer. The lysates were centrifuged at 10,000 xg for 20 min and clarified. Protein contents were quantified and separated by SDS- PAGE in $10 \%$ acrylamide gels and transferred to polyvinylidene difluoride membranes (Millipore, Billerica, MA), then immunoblotted with antibodies for the opioid receptors. Antibodies such as $\delta$-opioid receptor (DOR; sc-9111; 1:200 dilution), к-opioid receptor (KOR; sc-9112; 1:1000 dilution), and $\mu$-opioid receptor (MOR; sc-15310; 1:200 dilution) were purchased from Santa Cruz Biotechnology (Santa Cruz, CA). The bands were visualized using chemiluminescence Western Blotting Detection Reagents (Millipore) and quantified with ImageJ densitometry software (National Institutes of Health, Bethesda, MD). 


\section{Statistical analysis}

Data are expressed as means \pm SEM. Time response data are presented as PWT in g. Dose-response data are presented as \%MPE. Behavioral experiments were analyzed by repeated measures two-way analysis of variance (ANOVA) followed by a post-hoc test with Tukey's test for multiple comparisons. Values with $P<0.05$ were considered to be statistically significant as compared to vehicle treatment. Data of \%MPE were analyzed by ANOVA followed by a post-hoc test with Bonferroni correction. Values with $P<$ 0.01 were considered to be statistically significant as compared to vehicle treatment. One-way ANOVA followed by a post-hoc test with Tukey's test was performed for comparisons of densitometry between the groups.

\section{RESULTS}

1. Intrathecal SOG increased PWT in a dose-dependent manner

Incision of the hind paws significantly decreased PWT to the mechanical stimulation, but PWT at the contralateral side (non-incised paw) was not changed during stimula-

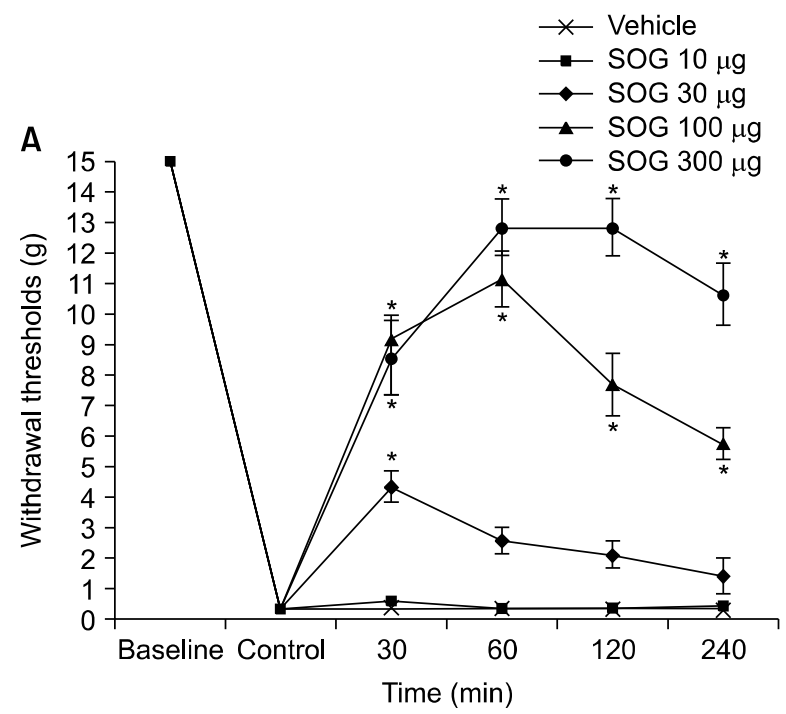

tion. The PWT decreased significantly $2 \mathrm{~h}$ after incision and intrathecal SOG showed a significant increase of the PWT at the ipsilateral paw in a dose-dependent fashion (Fig. 2A). Significant effects were shown from the dose of $30 \mu \mathrm{g}$ at $30 \mathrm{~min}$ after SOG administration (4.37 \pm 0.52 $\mathrm{g}$ ), but a constant effect was shown only in $100 \mu \mathrm{g}$ and $300 \mu \mathrm{g}$ throughout the observational periods $(* P<0.001$ compared to the vehicle). The maximum effect was achieved in a dose of $300 \mu \mathrm{g}$ at $60 \mathrm{~min}$ after intrathecal SOG administration $(12.85 \pm 0.93 \mathrm{~g})$.

\section{Maximal possible effects and ED50 of SOG}

Maximum effects of each dose were seen at $30 \mathrm{~min}$ after intrathecal administration of SOG in doses of $10 \mu \mathrm{g}$ and $30 \mu \mathrm{g}$, while at $60 \mathrm{~min}$ in doses of $100 \mu \mathrm{g}$ and $300 \mu \mathrm{g}$. Maximal possible effects were calculated with each dose of SOG (Fig. 2B). Intrathecal SOG administration showed a significant increase of \%MPE in a dose-dependent manner. The \%MPE in an intrathecal SOG administration dose of $300 \mu \mathrm{g}$ showed the greatest result, which was $83.35 \% \pm$ $6.35 \%$. The maximal effects of these individual doses were used for analysis of standard linear regression in order to calculate ED50 values (Fig. 3). The ED50 value of intra-

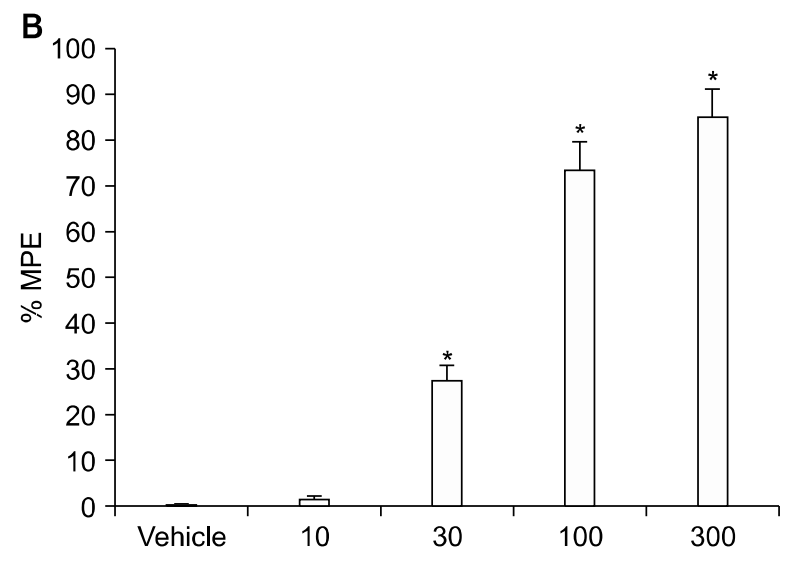

Fig. 2. (A) Effects of intrathecal sec-O-glucosylhamaudol (SOG) on paw withdrawal threshold (PWT) after incision. Data are presented as withdrawal thresholds $(\mathrm{g})$. Each value represents the mean \pm standard error of mean. (B) Maximal possible effects (MPE) of SOG according to the dose. Data are presented as \%MPE. Intrathecal SOG administration showed a significant increase of the PWT and \%MPE in a dose-dependent manner. Baseline is the withdrawal threshold measured before paw incision. Control is two hours after paw incision. Vehicle, $70 \%$ dimethyl sulfoxide. ${ }^{\star} P<0.001$ compared to the vehicle. 
thecal SOG was $191.3 \mu \mathrm{g}$ (95\% confidence intervals [CI], 102.3-357.8) with a slope of 50.74 (95\% CI, 33.55-67.94).

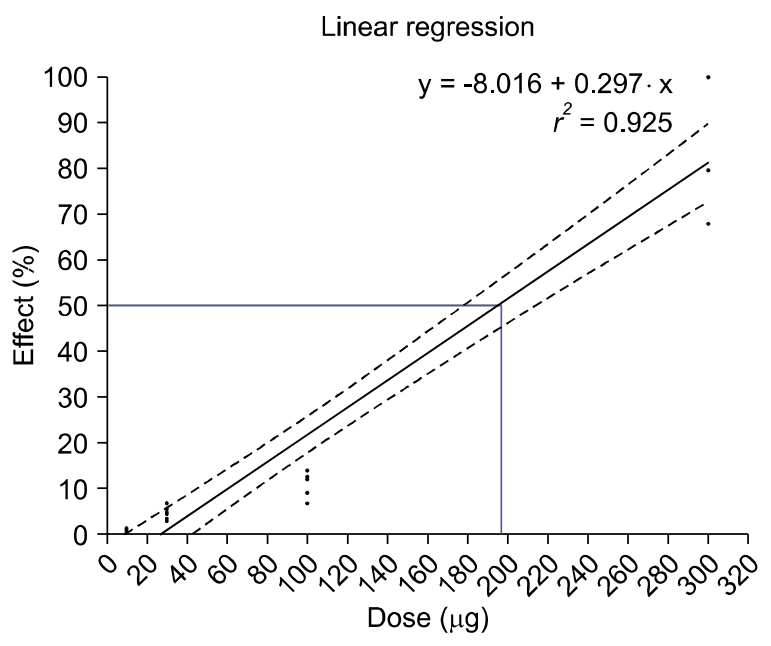

Fig. 3. Linear regression of the maximal effects of these individual doses of sec-O-glucosylhamaudol (SOG). The median effective analgesic dose of intrathecal SOG was $191.3 \mu \mathrm{g}$ (95\% confidence interval [Cl], 102.3-357.8) with a slope of $50.74(95 \% \mathrm{Cl}, 33.55-67.94)$.

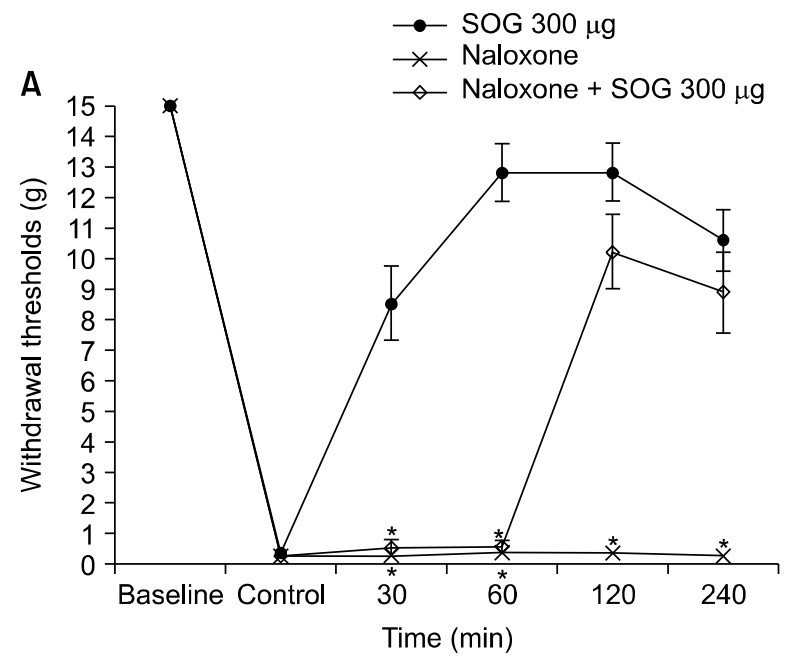

3. Opioid antagonist reversed the antinociceptive effect of intrathecal SOG

Intrathecal administration of naloxone, an opioid antagonist, which was administered $10 \mathrm{~min}$ before the delivery of SOG, reversed the anti-nociceptive effect of the SOG (Fig. $4 \mathrm{~A})$. Intrathecal administration of naloxone alone showed no antinociceptive effect after paw incision. Intrathecal administration of naloxone prior to the SOG $300 \mu \mathrm{g} \mathrm{de}-$ creased PWT $(0.82 \pm 0.52 \mathrm{~g})$, which resembles the effect of the vehicle alone until $60 \mathrm{~min}$, but the inhibitory effect of naloxone against SOG vanished at 120 min after paw incision. The maximal PWT of naloxone + SOG $300 \mu \mathrm{g}$ showed $11.17 \pm 0.81 \mathrm{~g}$ at $120 \mathrm{~min}$ after paw incision. which was similar to the effect of SOG $300 \mu \mathrm{g}$ alone. The \%MPE in the administration of naloxone before delivery of SOG $300 \mu \mathrm{g}$ was $3.50 \% \pm 3.50 \%$ at $60 \mathrm{~min}$ after incision, which was similar to the \%MPE of vehicle or naloxone alone (Fig. 4B). However, the \%MPE increased to $73.95 \% \pm$ $5.52 \%$ at $120 \mathrm{~min}$, which was similar to the effect of SOG $300 \mu \mathrm{g}$ alone.

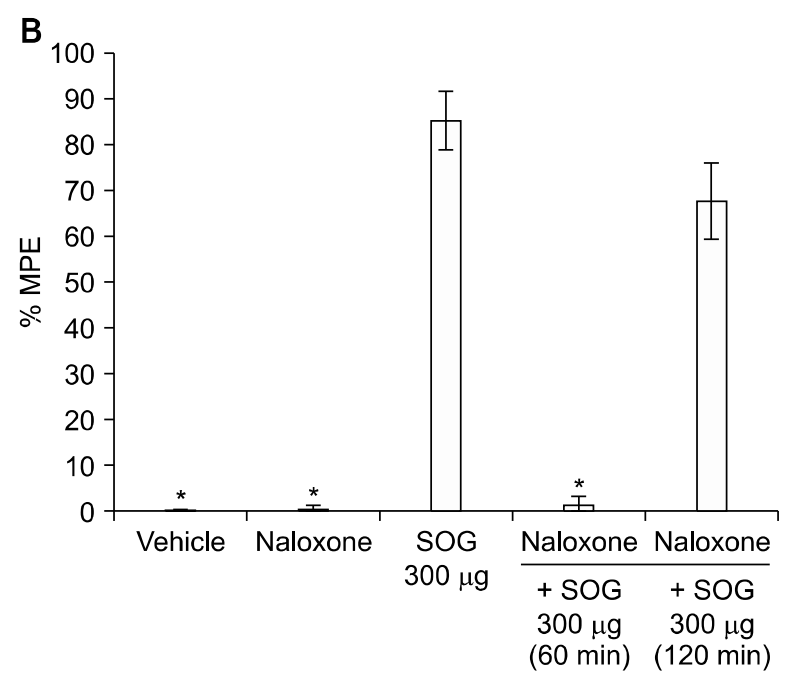

Fig. 4. (A) The inhibitory effects of intrathecal naloxone against sec-O-glucosylhamaudol (SOG). Data are presented as withdrawal thresholds $(\mathrm{g})$. Each value represents the mean \pm standard error of mean. (B) Maximal possible effects (MPE) of SOG according to the inhibitory effects of intrathecal naloxone. Data are presented as \%MPE. Intrathecal administration of naloxone alone showed no antinociceptive effect. Intrathecal administration of naloxone 10 min before the delivery of SOG $300 \mu \mathrm{g}$ decreased paw withdrawal threshold until $60 \mathrm{~min}$. The \%MPE in the administration of naloxone before delivery of SOG $300 \mu \mathrm{g}$ was decreased until $60 \mathrm{~min}$, which was similar to the \%MPE of vehicle or naloxone alone. The inhibitory effect of naloxone against SOG vanished at $120 \mathrm{~min}$. Baseline is the withdrawal threshold measured before paw incision. Control is two hours after paw incision. ${ }^{\star} P<0.001$ compared to the SOG $300 \mu \mathrm{g}$. 
A

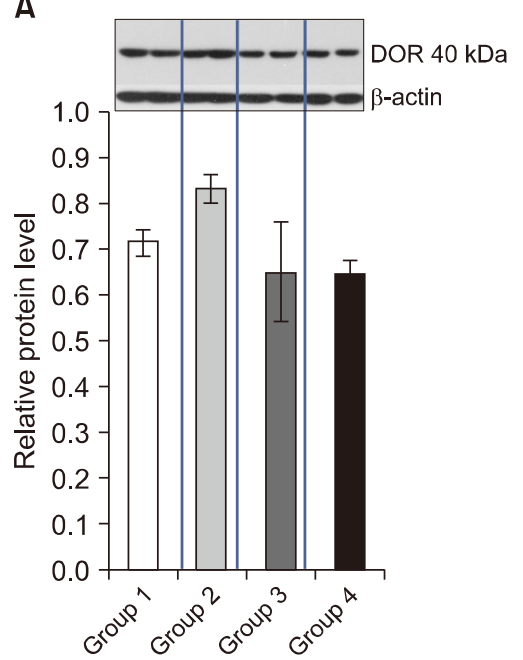

B

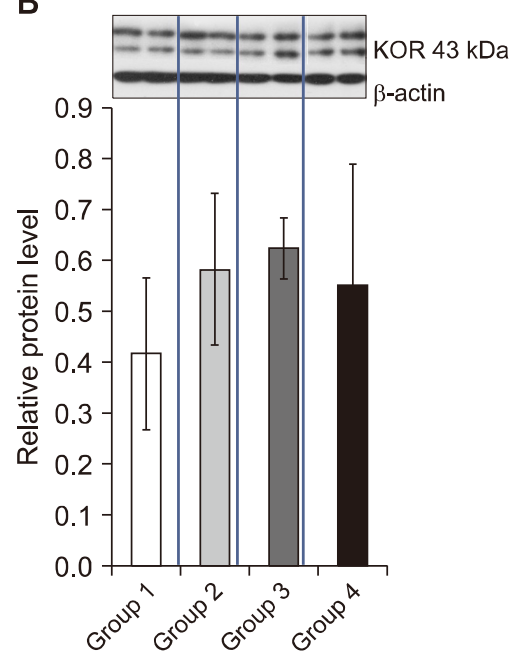

C

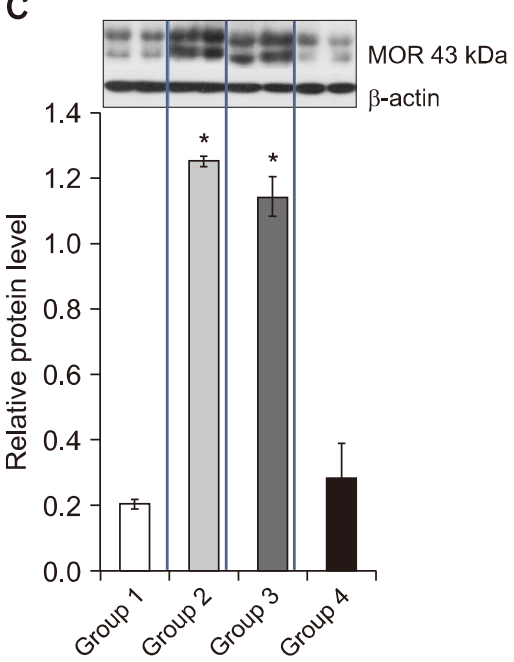

Fig. 5. Protein levels of (A) $\delta$-opioid receptor (DOR), (B) $\kappa$-opioid receptor (KOR), and (C) $\mu$-opioid receptor (MOR) in the spinal cord of rats. The protein levels of DOR and KOR showed no significant differences among the groups. Expression of MOR protein was increased by paw incision, and decreased by administration of sec-O-glucosylhamaudol (SOG). Data represent protein levels normalized to $\beta$-actin expression. Each value represents mean \pm standard error of mean of five rats. Group 1, naïve group; Group 2, control group (2 h after incision of left hind paw); Group 3, $1 \mathrm{~h}$ after vehicle administration (70\% dimethyl sulfoxide); Group 4, $1 \mathrm{~h}$ after SOG $300 \mu \mathrm{g}$ administration. ${ }^{\star} P<0.05$, compared with naïve group.

4. The protein levels of MOR were decreased by administration of SOG

The protein levels of $\mathrm{DOR}$ and $\mathrm{KOR}$ showed no significant differences among the groups (Fig. 5A, B). The protein levels of MOR were increased by paw incision compared to the naïve group and were not significantly altered by administration of $70 \%$ DMSO (Fig. 5C). However, administration of SOG decreased the protein levels of MOR, which showed no significant differences compared to the naïve group (Fig. $5 \mathrm{C}$ ).

\section{DISCUSSION}

Effective analgesia after surgery not only has significant physiological benefits, but is also one of the most important factors for patients [20]. The surgical incisions lead to the activation and sensitization of peripheral nociceptors and spinal dorsal horn neurons through the biological response of releasing proinflammatory mediators at an incision site, and lead to pain sensation [6,21]. Numerous studies on the pharmacological spinal modulation of the pain and allodynia after incision have been conducted [6]. However, opioids that act on the central and peripheral receptors are still the main analgesic agent for postoperative pain management, but have limitations in clinical use because of their side effects such as nausea, vomiting, pruritus, urinary retention, and respiratory depression [20]. Thus, there are ongoing attempts to develop analgesic agents with reduced side effects by targeting putative opioid receptor splice variants or the receptor heterooligomers to separate the analgesic effects from undesirable ones [22].

Searching for a natural product as a promising analgesic candidate can be a part of that effort [23]. There is no doubt that natural products have provided key leads for drug discovery and the search for novel natural products with interesting bioactivity is an ongoing exercise [24]. As the number of studies on natural products increases, some flavonoids from medicinal plants showed the possibility that they can be promising candidates for new natural analgesic drugs [23]. Flavonoids have received increasing attention because of their bioactivity and their potential as anti-inflammatory and analgesic drugs. Recently, there was an interesting report that showed that micronized flavonoid fractions, made of a flavonoid fraction, reduced the severity of pain and intramuscular analgesic requirement after hemorrhoidectomy [25]. 
As mentioned above, the Peucedanum japonicum Thunb. has been traditionally used as an analgesic for headaches or neuralgic disease [8,9]. Substances derived from Peucedanum species show effects such as antiplatelet aggregation, antioxidant activity, anti-inflammatory activity, and $\mathrm{COX}-1$ and $\mathrm{COX}-2$ inhibition [9-12] which are the same bioactivity as chromone derivatives [24]. Chromones (4H-chomen-4-ones) are a group of naturally occurring compounds ubiquitous in nature, particularly in plants [26]. Classification in terms of chromone and flavonoid alkaloids is based on the part of the molecule to which the nitrogenous moiety is attached. Species of Peucedanum have constituents of coumarins, oils, chromones, flavonoids, and other acids [27]. SOG, one of the constituents of Peucedanum species, is known as a new chromone belonging to the flavonoids family [28], which has analgesic and antiallodynic activity [12-14].

The mechanism of analgesic activity in SOG is still unknown because there are only few studies on the effects of SOG. Zheng et al. [12] isolated compounds from the Peucedanum japonicum Thunb. and revealed that SOG has an inhibitory effect on COX-1 and COX-2 by COX inhibition assay, but that SOG showed only a weak effect. Okuyama et al. [13] showed that oral administration of SOG $80 \mathrm{mg} / \mathrm{kg}$ significantly increased pain threshold in the tail pressure examination and in the test to detect neuropathic pain responses (modified Randall \& Selitto test). They suggested that the effect of SOG acts on the opioid receptor of the central nervous system because the analgesic effect of SOG was reversed by the injection of naloxone. In particular, a recent study showed that intrathecal administration of SOG had an antinociceptive effect in a formalin test of a rat model and the possibility of the involvement of SOG on opioid receptors [14]. These results of previous studies are in agreement with our results. In the present study, intrathecal SOG showed a strong antinociceptive effect for pain after incision in a dose-dependent manner. The maximal analgesic effect was achieved in a dose of $300 \mu \mathrm{g}$ at $60 \mathrm{~min}$ after intrathecal SOG administration $(12.85 \pm 0.93 \mathrm{~g})$ and the \%MPE was $83.35 \% \pm 6.35 \%$.

We hypothesized the potential mechanisms of the antinociceptive effect of SOG according to the effect of flavonoids. First, SOG may acts as an anti-inflammatory drug on the central nervous system. There are numerous studies on the anti-inflammatory effect of flavonoids, with many particularly focused on the inhibitory activities to- ward COX -1 and COX $-2[23,26]$. Thus, the COX inhibitory effect of flavonoids has been used as a basis for the synthesis of new anti-inflammatory agents [29]. One study on the chromone extract Saposhnikovia divaricata, which has SOG as one of its components, showed that it possesses potential anti-inflammatory, anti-osteoarthritis, and anti-rheumatoid arthritis effects [30]. However, SOG showed only a weak inhibitory effect on both $\mathrm{COX}-1$ and $\mathrm{COX}-2$ according to the study of Zheng et al. [12]. Thus, the connection between the antinociceptive effect of SOG on incisional pain and the inhibition of COX is small. However, anti-inflammatory actions related to the lipoxygenase pathway or nitric oxide production inhibitors should be evaluated in the future [26]. Second, the antinociceptive effects of SOG are closely associated with opioid-related mechanisms themselves. Mechanisms of pain caused by a surgical incision are complex, but it is obvious that peripheral inflammation and incision contribute to postoperative pain and incision-induced pain behavior, and that exogenous opioids modulate these effects [6].

Mechanisms of postoperative pain in the plantar incision model are related with both neuropathic and inflammatory components [31]. The increase of MOR expression is associated with inflammatory components, whereas the increase in DOR expression is associated with neuropathic components [31]. In the present study, the maximal antinociceptive effect of SOG was significantly reverted by naloxone, which was administered $10 \mathrm{~min}$ before the delivery of SOG. Moreover, the reverse effect lasted only about $1 \mathrm{~h}$, which is in accord with the blocking effect of the intrathecal naloxone on the drugs which have an antinociceptive effect in the involvement of the opioid receptor [32]. According to the western blotting analysis of the current study, expression of MOR protein was increased by paw incision and administration of SOG decreased the protein levels of MOR. However, expression of $\mathrm{DOR}$ and $\mathrm{KOR}$ showed no significant differences among the groups. These results suggest that the antinociceptive effects of SOG are related with opioid mechanisms, especially with MOR. Several studies have shown that flavonoids activate opioids system because of their structure-activity relationship with opioid receptors [33,34]. The actions of 3,3-dibromoflavanone, synthetic flavonoids, bind to MOR in the central nervous system and produce antinociception [34]. According to the literature, flavonoids act as, and are, opioid receptor ligands, and the stereo- 
chemistry of the $\mathrm{C} 2$ and $\mathrm{C} 3$ positions is important for antagonist activity and selectivity, thus structural modifications to the core structure can modulate intrinsic activity at opioid receptors [33]. Given these factors, further research into SOG could be a novel structural scaffold for the development of new drugs targeting opioid receptors that only work on pain and avoid adverse effects.

There are some limitations to this study. First, DMSO itself can have an antinociceptive effect [35]. Because SOG was hydrophobic substances, DMSO had to be used as a solvent. However, 70\% DMSO alone showed no antinociceptive effect. Second, further molecular work is needed to prove the hypothetic mechanisms of SOG. Further detailed evaluations about the anti-inflammatory effect and structural effect on opioid receptors should be done. Third, further evaluations of the side-effects of SOG are needed for the clinical application, because of its relationship with opioid receptors.

So far, the information about SOG on the antinociceptive effect and pharmacological mechanisms has been very limited. Therefore, we evaluated the potential effect of SOG on postoperative pain. In summary, intrathecal administration of SOG significantly decreased PWT in a dose-dependent manner, and the maximal antinociceptive effects of SOG were significantly reverted until $60 \mathrm{~min}$ by naloxone. Intrathecal SOG decreased the expression of MOR after paw incision.

In conclusion, these results strongly suggest that intrathecal SOG showed significant antinociceptive effect on the postoperative pain model through the involvement of MOR. The current study can be a foundation for the development of new analgesics for postoperative pain control based on natural products such as SOG.

\section{CONFLICT OF INTEREST}

No potential conflict of interest relevant to this article was reported.

\section{REFERENCES}

1. Pyati S, Gan TJ. Perioperative pain management. CNS Drugs 2007; 21: 185-211.

2. Kang S, Brennan TJ. Mechanisms of postoperative pain. Anesth Pain Med 2016; 11: 236-48.

3. Shin DJ, Yoon MH, Lee HG, Kim WM, Park BY, Kim YO, et al. The effect of treatment with intrathecal ginsenosides in a rat model of postoperative pain. Korean J Pain 2007; 20: 100-5.

4. Kim IJ, Park CH, Lee SH, Yoon MH. The role of spinal adrenergic receptors on the antinociception of ginsenosides in a rat postoperative pain model. Korean $J$ Anesthesiol 2013; 65: 55-60.

5. Yeager MP, Glass DD, Neff RK, Brinck-Johnsen T. Epidural anesthesia and analgesia in high-risk surgical patients. Anesthesiology 1987; 66: 729-36.

6. Pogatzki-Zahn EM, Segelcke D, Schug SA. Postoperative pain-from mechanisms to treatment. Pain Rep 2017; 2: e588.

7. McCurdy CR, Scully SS. Analgesic substances derived from natural products (natureceuticals). Life Sci 2005; 78: 47684.

8. Ikeshiro Y, Mase I, Tomia Y. Dihydropyranocoumarins from roots of Peucedanum japonicum. Phytochemistry 1992; 31 : 4303-6.

9. Chen IS, Chang CT, Sheen WS, Teng CM, Tsai IL, Duh CY, et al. Coumarins and antiplatelet aggregation constituents from Formosan Peucedanum japonicum. Phytochemistry 1996; 41: 525-30.

10. Hisamoto M, Kikuzaki H, Ohigashi H, Nakatani N. Antioxidant compounds from the leaves of Peucedanum japonicum thunb. J Agric Food Chem 2003; 51: 5255-61.

11. Zimecki M, Artym J, Cisowski W, Mazol I, Wlodarczyk M, Gleńsk M. Immunomodulatory and anti-inflammatory activity of selected osthole derivatives. Z Naturforsch C 2009; 64: 361-8.

12. Zheng MS, Jin WY, Son KH, Chang HW, Kim HP, Bae KH, et al. The constituents isolated from Peucedanum japonicum Thunb. and their Cyclooxygenase (COX) inhibitory activity. Korean J Med Crop Sci 2005; 13: 75-9.

13. Okuyama E, Hasegawa T, Matsushita T, Fujimoto H, Ishibashi M, Yamazaki M. Analgesic components of saposhnikovia root (Saposhnikovia divaricata). Chem Pharm Bull (Tokyo) 2001; 49: 154-60.

14. Kim SH, Jong HS, Yoon MH, Oh SH, Jung KT. Antinociceptive effect of intrathecal sec-O-glucosylhamaudol on the formalininduced pain in rats. Korean J Pain 2017; 30: 98-103.

15. Zimmermann M. Ethical guidelines for investigations of experimental pain in conscious animals. Pain 1983; 16: 109-10.

16. Yaksh TL, Rudy TA. Chronic catheterization of the spinal subarachnoid space. Physiol Behav 1976; 17: 1031-6.

17. Brennan TJ, Vandermeulen EP, Gebhart GF. Characterization of a rat model of incisional pain. Pain 1996; 64: 493-501.

18. Chaplan SR, Bach FW, Pogrel JW, Chung JM, Yaksh TL. Quantitative assessment of tactile allodynia in the rat paw. J Neurosci Methods 1994; 53: 55-63.

19. Tallarida RJ, Murray RB. Manual of pharmacologic calcu- 
lations with computer programs. 2nd ed. New York, SpringerVerlag. 1987, pp 1-95.

20. Garimella V, Cellini C. Postoperative pain control. Clin Colon Rectal Surg 2013; 26: 191-6.

21. Woolf CJ, Chong MS. Preemptive analgesia: treating postoperative pain by preventing the establishment of central sensitization. Anesth Analg 1993; 77: 362-79.

22. Law PY, Reggio PH, Loh HH. Opioid receptors: toward separation of analgesic from undesirable effects. Trends Biochem Sci 2013; 38: 275-82.

23. Xiao X, Wang X, Gui X, Chen L, Huang B. Natural flavonoids as promising analgesic candidates: a systematic review. Chem Biodivers 2016; 13: 1427-40.

24. Khadem S, Marles RJ. Chromone and flavonoid alkaloids: occurrence and bioactivity. Molecules 2011; 17: 191-206.

25. Colak T, Akca T, Dirlik M, Kanik A, Dag A, Aydin S. Micronized flavonoids in pain control after hemorrhoidectomy: a prospective randomized controlled study. Surg Today 2003; 33: 828-32.

26. Silva CF, Pinto DC, Silva AM. Chromones: a promising ring system for new anti-inflammatory drugs. ChemMedChem 2016; 11: 2252-60.

27. Sarkhail P. Traditional uses, phytochemistry and pharmacological properties of the genus Peucedanum: a review. J Ethnopharmacol 2014; 156: 235-70.

28. Sasaki H, Taguchi H, Endo T, Yosioka I. The constituents of Ledebouriella seseloides WOLFF. I. Structures of three new chromones. Chem Pharm Bull (Tokyo) 1982; 30: 3555-62.

29. Gautam R, Jachak SM, Kumar V, Mohan CG. Synthesis, biological evaluation and molecular docking studies of stellatin derivatives as cyclooxygenase (COX-1, COX-2) inhibitors and anti-inflammatory agents. Bioorg Med Chem Lett 2011; 21: 1612-6.

30. Chun JM, Kim HS, Lee AY, Kim SH, Kim HK. Antiinflammatory and antiosteoarthritis effects of saposhnikovia divaricata ethanol extract: in vitro and in vivo studies. Evid Based Complement Alternat Med 2016; 2016: 1984238.

31. de Oliveira Junior JO, de Freitas MF, Bullara de Andrade C, Chacur M, Ashmawi HA. Local analgesic effect of tramadol is mediated by opioid receptors in late postoperative pain after plantar incision in rats. J Pain Res 2016; 9: 797-802.

32. Berger $\vee$, Alloui A, Kemeny JL, Dubray C, Eschalier A, Lavarenne J. Evidence for a role for bulbospinal pathways in the spinal antinociceptive effect of systemically administered vapreotide in normal rats. Fundam Clin Pharmacol 1998; 12: 200-4.

33. Katavic PL, Lamb K, Navarro H, Prisinzano TE. Flavonoids as opioid receptor ligands: identification and preliminary structure-activity relationships. J Nat Prod 2007; 70: 127882.

34. Higgs J, Wasowski C, Loscalzo LM, Marder M. In vitro binding affinities of a series of flavonoids for $\mu$-opioid receptors. Antinociceptive effect of the synthetic flavonoid 3,3-dibromoflavanone in mice. Neuropharmacology 2013; 72: 9-19.

35. Colucci M, Maione F, Bonito MC, Piscopo A, Di Giannuario A, Pieretti S. New insights of dimethyl sulphoxide effects (DMSO) on experimental in vivo models of nociception and inflammation. Pharmacol Res 2008; 57: 419-25. 